\title{
CONDITIONS ON BANACH ALGEBRAS WHICH IMPLY FINITE DIMENSIONALITY
}

\author{
by ALAN W. TULLO \\ (Received 24th June 1974)
}

1

The purpose of this note is to describe some algebraic conditions on a Banach algebra which force it to be finite dimensional. We shall assume throughout that we are dealing with Banach algebras over the field of complex numbers, $\mathbf{C}$.

Our main result is Theorem 5 which states that a semi-prime Banach algebra which coincides with its socle is necessarily finite dimensional. Most of the known results on algebraic conditions which force a Banach algebra to be finite dimensional depend very heavily on the supply of idempotents in the algebra. It is therefore to be expected that these results will be related in some way to Theorem 5. In fact, many may be deduced from our result and as an example we prove a lemma of Kaplansky (5, Lemma 7).

\section{2}

Our first lemma is a special case of a result on stability of bases (see for example (8, Chapter 1 , Theorem 9.1)) so we give here only a sketch of the proof.

Lemma 1. Let $X$ be a normed linear space of dimension at least $n$ and let $x_{1}, \ldots, x_{n}$ be any $n$ linearly independent vectors in $X$. There is an $\varepsilon>0$ such that if the $n$ vectors $y_{1}, \ldots, y_{n}$ satisfy $\left\|x_{i}-y_{i}\right\|<\varepsilon(i=1, \ldots, n)$ then $y_{1}, \ldots, y_{n}$ are also linearly independent.

Proof. By making use of the compactness of $\left\{\alpha \in \mathbf{C}^{n}:\|\alpha\|=1\right\}$ we may show that $\left\{x \in X^{n}: \exists \alpha \in \mathbf{C}^{n} \backslash\{0\}\right.$ such that $\left.\sum_{i=1}^{n} \alpha_{i} x_{i}=0\right\}$ is closed. The result now follows.

Definition. Given a normed linear space $X$, we write $B(X)$ for the normed linear space of all bounded linear operators on $X$. For any operator $T \in B(X)$, rank $(T)$ is the dimension of the range space of $T$.

Lemma 2. Let $X$ be a normed linear space. For each positive integer $n_{0}$ the set $X_{n_{0}}=\left\{T \in B(X): \operatorname{rank}(T) \leqq n_{0}\right\}$ is closed.

Proof. Suppose $\left\{T_{k}\right\} \subset X_{n_{0}}, T_{k} \rightarrow T$ in the uniform norm on $B(X)$ and rank $(T)>n_{0}$. Choose $n_{0}+1$ linearly independent vectors $y_{j}=T x_{j}$ in $T(X)\left(j=1, \ldots, n_{0}+1\right)$. By Lemma 1 , there is an $\varepsilon>0$ such that if $\left\|y_{j}-z_{j}\right\|<\varepsilon\left(j=1, \ldots, n_{0}+1\right)$ then $z_{1}, \ldots, z_{n_{0}+1}$ are linearly independent.

$$
\text { E.M.S. }-20 / 1-A
$$


Since $T_{k} \rightarrow T$ we have $\left\|T_{k} x_{j}-y_{j}\right\|<\varepsilon\left(j=1, \ldots, n_{0}+1\right)$ for large enough $k$. This contradicts rank $\left(T_{k}\right) \leqq n_{0}$.

3

At this point it is convenient to give some definitions and results which will be required in what follows.

An algebra, $A$ is (topologically) simple if it contains no proper (closed) twosided ideals; it is semi-prime if the only left ideal in $A$ which has square zero is the zero ideal. If $A$ contains minimal left (right) ideals, the sum of all the minimal left (right) ideals is called the left (right) socle of $A$. If both socles exist and are equal, the resulting two-sided ideal is called simply the socle of $A$ and is denoted by $\operatorname{soc}(A)$. We recall that when $A$ is semi-prime and contains minimal one-sided ideals soc $(A)$ exists.

Lemma 3. A simple Banach algebra with minimal one-sided ideals is finite dimensional.

Proof. Let $A$ be a simple Banach algebra and let $L$ be a minimal left ideal of $A$.

If $A L=\{0\}$ then $\{x \in A: A x=\{0\}\}=A$ so that $A^{2}=\{0\}$. Thus every subspace of $A$ is a two-sided ideal and so $A$ must be one-dimensional or $\{0\}$.

Now suppose $A L \neq\{0\}$. Then the left regular representation of $A$ on $L$ is faithful and irreducible so that $A$ can be regarded as a strictly dense Banach algebra of operators on $L$ (7, Theorem (2.4.6)). In particular, $A$ is semi-simple so that $\operatorname{soc}(A)$ is defined and $A=\operatorname{soc}(A)$. Thus $A$ consists of finite rank operators $(7$, p. 65$)$. Let $A_{n}=\{T \in A: \operatorname{rank}(T) \leqq n\}$. By Lemma $2, A_{n}$ is closed in the uniform operator topology and hence is closed in the given norm on $A$. Now $A=\bigcup_{n=1}^{\infty} A_{n}$ so, by Baire's category theorem, $A_{n_{0}}$ has non-empty interior for some integer $n_{0}$. Thus there is $T_{0} \in A_{n_{0}}$ and $\varepsilon>0$ such that rank $(T) \leqq n_{0}$ whenever $\left\|T-T_{0}\right\|<\varepsilon$. It follows that rank $(T) \leqq 2 n_{0}$ for each $T \in A$ and hence that $A$ is finite dimensional.

Notice that the Calkin algebra on an infinite dimensional separable Hilbert space is an infinite dimensional simple Banach algebra which has no minimal one-sided ideals.

Proposition 4. A topologically simple Banach algebra which has a unit and contains minimal one-sided ideals is finite dimensional.

Proof. Let $A$ be a topologically simple Banach algebra with unit, 1 , and let $L$ be a minimal left ideal of $A$. As in Lemma 3 , we may regard $A$ as a strictly dense Banach algebra of operators on $L$. Since $\operatorname{soc}(A)$ is dense in $A, 1$ is a limit of finite rank operators in the norm on $A$ and hence in the uniform operator topology. The ideal $L$ is closed in the given norm on $A$ and so the compact 
operators on $L$ form a uniformly closed set. Thus 1 is a compact operator and hence $L$ is finite dimensional. The result follows since $A \subset B(L)$.

Notice that the compact operators on an infinite dimensional Hilbert space form an infinite dimensional topologically simple Banach algebra which contains minimal one-sided ideals but has no unit.

Theorem 5. If $A$ is a semi-prime Banach algebra with minimal one-sided ideals and $A=\operatorname{soc}(A)$ then $A$ is finite dimensional.

Proof. Since $A$ is semi-prime, a left idea of $A$ is minimal if and only if it is irreducible as a left $A$-module.

Let $L$ be a minimal left ideal of $A$. The sum of the minimal left ideals of $A$ which are module isomorphic to $L$ is called the homogeneous component of soc $(A)$ corresponding to $L$. The fact that $A$ is semi-prime implies that $A$ $(=\operatorname{soc}(A))$ is a direct sum of its homogeneous components and that these are simple algebras (see (2), pp. 64, 65).

Distinct homogeneous components have product $\{0\}$ and minimal left ideals in $A$ are of the form $A e$ for some minimal idempotent $e \in A$. Thus if the set of distinct homogeneous components is infinite we can construct an infinite orthogonal sequence, $\left\{e_{n}\right\}$, of (minimal) idempotents. Let

$$
x=\sum_{n=1}^{\infty} 2^{-n}\left\|e_{n}\right\|^{-1} e_{n}
$$

Then $x \in A \mid \operatorname{soc}(A)$ which contradicts $A=\operatorname{soc}(A)$. Suppose $H_{1}, \ldots, H_{n}$ are the distinct homogeneous components of $A$ so that $A=H_{1} \oplus \ldots \oplus H_{n}$. Each $H_{k}$ is closed since it is the annihilator of the direct sum of the rest of the $H_{j}$ 's. So, each $H_{k}$ is a simple Banach algebra with minimal left ideals. An application of Lemma 3 concludes the proof.

Corollary 6. If $A$ is a Banach algebra satisfying $A=\operatorname{soc}(A)$ then the (Jacobson) radical of $A$ has finite codimension in $A$.

Notice that we cannot in general relax the condition $A=\operatorname{soc}(A)$; for example any infinite dimensional semi-simple annihilator Banach algebra has dense socle. However we do have the following theorem:

Theorem 7. Let $A$ be a semi-simple Banach algebra with unit and suppose that $A$ has dense socle. Then $A$ is finite dimensional.

Proof. Alexander (1, Theorem 7.3) has shown that any semi-simple Banach algebra which has a dense socle is a compact Banach algebra. That is, $x \rightarrow a x a$ is a compact operator on $A$ for each $a \in A$. The result is thus immediate since $1 \in A$.

4

We now give the promised proof of Kaplansky's lemma. In the following, if $x$ is an element of an algebra $B$, we write $\operatorname{Sp}(B, x)$ for the spectrum of $x$ relative to $B$. 
Lemma (5, Lemma 7). Let $A$ be a semi-simple Banach algebra in which each element has a finite spectrum. Then $A$ is finite dimensional.

Proof. First note that an orthogonal sequence of idempotents in $A$ is necessarily finite. For, if $\left\{e_{n}\right\}$ is such a sequence and $\left\{e_{n}\right\}$ is infinite, we may choose a sequence $\left\{c_{n}\right\}$ of scalars such that $x=\sum_{n=1}^{\infty} c_{n} e_{n}$ is in $A$ and such that there are an infinite number of distinct $c_{n}$ 's. Since $x e_{n}=c_{n} e_{n}$, for each $n$, it follows that $\left\{c_{n}\right\}$ is contained in $\operatorname{Sp}(A, x)$.

If the spectrum of every element of $A$ is a singleton then $A$ is one-dimensional (5, Lemma 4). Otherwise $A$ contains a proper idempotent $e$. The Banach algebra $e A e$ is semi-simple and $\operatorname{Sp}(e A e, x)=\operatorname{Sp}(A, x)$ for each $x \in e A e$. If $e A e$ is not one dimensional we can find an idempotent $f \in e A e$ such that $0 \neq f \neq e$ and $f, e-f$ are orthogonal. This, together with the fact that any orthogonal sequence of idempotents must be finite, allows us to infer the existence of minimal idempotents in $A$.

Suppose $F=\left\{f_{1}, \ldots, f_{k}\right\}$ is a maximal orthogonal family of minimal idempotents of $A$. Let $f=f_{1}+\ldots+f_{k}$ and $B=(1-f) A(1-f)$. Then $B$ is semisimple and $\operatorname{Sp}(B, b)$ is finite for each $b \in B$. Since any idempotent in $B$ is orthogonal to $F$ it must be that $B=\{0\}$. Hence $A(1-f)$ and $(1-f) A$ have square zero and so, since $A$ is semi-simple, are zero. Thus $f=f_{1}+\ldots+f_{k}$ is identity for $A$ and $A=\operatorname{soc}(A)$. The result follows by Theorem 5 .

Remark. If in the above lemma we suppose that $A$ is a $B^{*}$-algebra then the conclusion still holds if we assume only that each self-adjoint element of $A$ has a finite spectrum (6, Theorem 1$)$. Theorem 5 can be used to give an elementary proof of this fact.

Definition. Let $A$ be any algebra.

(i) $A$ is (von Neumann) regular if for each $x \in A$ there is $y \in A$ such that

$$
x y x=x .
$$

(ii) $A$ is $\pi$-regular if for each $x \in A$ there is $y \in A$ and an integer $k$ such that

$$
x^{k} y x^{k}=x^{k}
$$

(iii) $A$ satisfies the descending chain condition (dcc) on principal left ideals if $A$ contains no properly decreasing infinite chains of principal left ideals.

Theorem 5 can be used to prove that regular Banach algebras (3, p. 58), semisimple $\pi$-regular Banach algebras (4, Remark (a), p. 63) and semi-simple Banach algebras which satisfy the dcc on principal left ideals are finite dimensional. A consequence of the last of these results is that any semi-simple Artinian Banach algebra is finite dimensional. ( $A$ is Artinian if it satisfies the dcc on left ideals.) 


\section{REFERENCES}

(1) J. C. AleXander, Compact Banach algebras, Proc. London Math. Soc. (3) 18 (1968), 1-18.

(2) N. JacoBson, Structure of rings (American Math. Soc. Coll. Publ. No. 37, Providence, 1956). 57-62.

(3) I. KaplansKy, Regular Banach algebras, J. Indian Math. Soc. 12 (1948),

(4) I. Kaplansky, Topological representations of algebras II, Trans. Amer. Math. Soc. 68 (1950), 62-75.

(5) I. Kaplansky, Ring isomorphisms of Banach algebras, Canadian J. Math. 6 (1954), 374-381.

(6) T. Ogasawara, Finite dimensionality of certain Banach algebras, J. Sci. Hiroshima Univ. Ser. A 17 (1951), 359-364.

(7) C. E. RICKART, General theory of Banach algebras (van Nostrand, 1960).

(8) I. Singer, Bases in Banach spaces I (Grundlehren der Math., Bd. 154, Springer, Berlin-Heidelberg-New York, 1970).

Mathematical Institute

EDINBURGH EH1 $1 \mathrm{HZ}$ 